\title{
Finding What Works: \\ Leadership Competencies for the Changing Healthcare Environment
}

\author{
Ann M. Herd \\ Assistant Professor \\ University of Louisville \\ College of Education and Human Development \\ Brittany L. Adams-Pope \\ Assistant Professor \\ University of Louisville \\ College of Business \\ Amanda Bowers \\ Graduate Student \\ University of Louisville \\ College of Education and Human Development \\ Brittany Sims \\ Graduate Student \\ University of Louisville \\ College of Business
}

\begin{abstract}
As the world of healthcare changes rapidly, healthcare leaders and managers must hone their leadership competencies in order to remain effective in their organizations. With changes such as the Affordable Care Act, increasing medical school costs, decreased graduation rates, and increased needs for care, how are current and future healthcare leaders adapting? In light of the large-scale changes in the healthcare field in recent years, the purpose of this study was to investigate which National Center for Healthcare Leadership (NCHL) competencies were referenced by exemplary healthcare leaders as most important for success in today's changing healthcare environment. Interviews were conducted with 26 mid- and upper-level healthcare leaders identified by the C-level executives in their organizations as exemplary performers. Change leadership, self-development, talent development, and team leadership were the top four NCHL competencies most frequently referenced, with thematic analysis revealing additional underlying themes in the exemplary leaders' dialogue.
\end{abstract}

\section{Introduction and Literature Review}

In the constantly changing world of healthcare, are healthcare leaders adapting to the "new normal" of dwindling resources, higher expectations, shortages of trained workers, and the overall "do more with less" requirements? Much has been written about the evidence-based 
approach to healthcare management and the competencies that accompany this approach (Calhoun, Dollett, Sinioris, Wainio, Butler, Griffith, \& Warden, 2008; Kovner, 2001; Kovner \& Rundall, 2006; Stefl, 2008), but how has the healthcare field evolved in terms of the competencies most needed for success as the industry has changed? One of the most popular healthcare leadership competency models in use today is the National Center for Healthcare Leadership (NCHL) model, which was designed based on extensive research nearly a decade ago (Calhoun, Dollett, Sinioris, Wainio, Butler, Griffith, \& Warden, 2008). While the competencies outlined in the model are acknowledged by experts to be robust (Calhoun et al., 2008; Garman \& Scribner, 2011), the healthcare landscape has changed significantly in recent years (Franco \& Almeida, 2011). Given the pervasive use of competency models, such as NCHL, used by healthcare organizations and educational institutions for a variety of human resource development and educational purposes (Calhoun, Vincent, Calhoun, \& Brandsen, 2008; Campion, Fink, Ruggeberg, Carr, Phillips, \& Odman, 2011; Clark \& Armit, 2010; Kovner, 2001; Kovner \& Rundall, 2006; McAlearney, 2010; Stefl \& Bontempo, 2008), thoughtful inquiry is needed to explore the leadership competencies most needed for effective performance in the current healthcare environment.

NCHL Healthcare Leadership Competency Model. Since the turn of the century, there has been a growing need for an improvement in the quality of health care in the United States. A 2003, Institute of Medicine (IOM), report Health Professions Education: A Bridge to Quality argued that the desired standard of care could not be reached without major reform of education and professional development across all sectors of health care. The report called for the creation of a core set of competencies that were broad enough to span across all segments of the health profession and would ultimately improve the overall quality of U.S. health care (IOM, 2003).

The National Center for Healthcare Leadership (NCHL, 2004) used a research-based competency modeling process to design a competency model delineating 26 competencies identified in the study as critical for healthcare leadership effectiveness (Calhoun et al., 2008). The 26 competencies are organized into the categories of transformation, execution, and people, as depicted in Figure 1. 


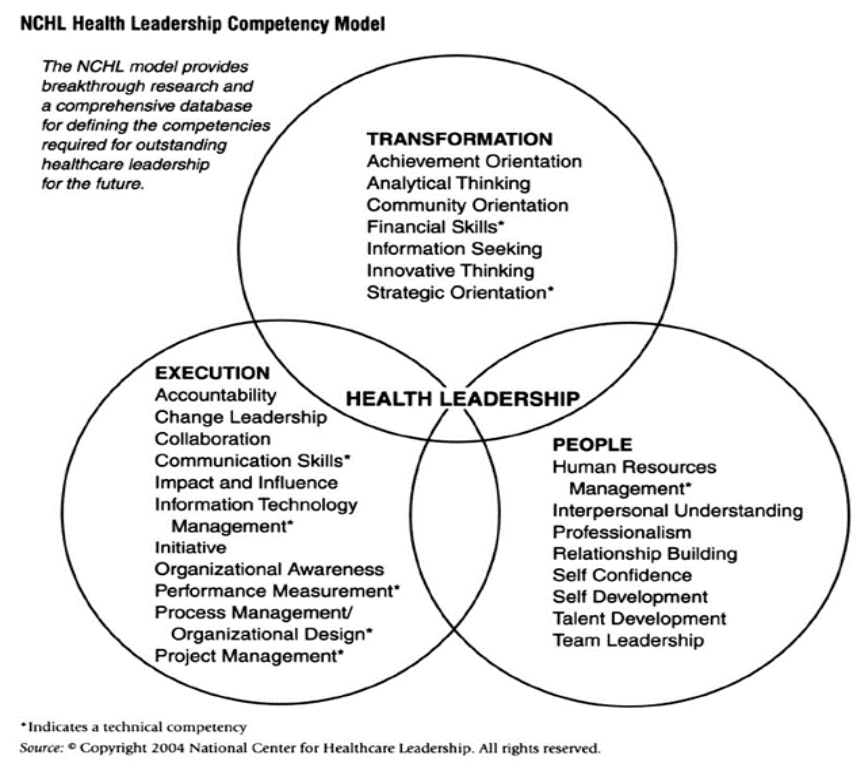

Figure 1. NCHL Healthcare Leadership Competency Model (www.nchl.org)

The model has gained recognition and use across a variety of healthcare organizations and educational settings (Clark \& Armit, 2010; Fried \& Fottler, 2013). While some organizations have used evidence-based research practices to determine the weighting of competencies most important for their organization's work, many organizations use all the competencies identified in the model, equally weighted, for a variety of integral HR purposes (Fried \& Fottler, 2013). Since the model's design over a decade ago, the healthcare landscape has changed significantly.

Changes in the Healthcare Field. A study published in 2011 by the Center for Creative Leadership (CCL) describes changes in the healthcare field that have had critical implications for leadership effectiveness. The study authors analyzed leadership effectiveness data from over 30,000 people working in the healthcare field, and state that, "for decades, US-based healthcare providers...have been operating in a shifting landscape. Advances in technology and new standards of care, new business models, a growing population and changing demographics have propelled ongoing change in the healthcare sector" (Patterson, Champion, Browning, Torain, Harrison, Gurvis, Fleenor, \& Campbell, 2011, p. 3). In light of these changes, key findings and recommendations derived from this study include the need for increased opportunities for broad cross-functional and cross-organizational learning opportunities for healthcare leaders, as well as opportunities for the development of team leadership skills (Patterson et al., 2011).

Healthcare organizations in particular need to be "learning organizations" in order to keep up with the immense amount and rapid pace of changes occurring at all levels and in all areas of the organizations' processes (Franco \& Almedia, 2011). Conceptualizations of learning organizations suggest that they build a culture of continuous learning among their members, thus encouraging perpetual transformation and adaptations to change (Castells, 2001; Schon, 1991; Senge, 1990). Learning organizations "intentionally develop strategies to promote learning" (Franco \& Almedia, 2011, p. 785). With regard to healthcare leadership competencies, leadership styles in relation to the organizational learning characteristics needed in today's 
healthcare organizations have received relatively little attention (Franco \& Almedia, 2011).

In light of the pervasive use of the NCHL healthcare competency model as well as the growing recognition that healthcare organizations need to adapt to a rapidly changing environment, the purpose of the present study was to address the research question: How do the competencies described by current healthcare leaders as most necessary for leading in today's healthcare environment compare to the competencies in the NCHL (Calhoun et al., 2008) model? A qualitative methodology investigating exemplary leaders' accounts relating to their performance as healthcare leaders was deemed as most appropriate for answering this research question, and is described below.

\section{Method}

Sample and Procedures. A qualitative research method was used to answer the research question (Berg, Lune, \& Lune, 2004; DiCicco-Bloom \& Crabtree, 2006) in order to provide the researchers with contextual insights into the daily working lives of healthcare leaders in their organizations. The 26 participants in this study were exemplary healthcare leaders as identified by $15 \mathrm{C}$-level executives who participated by invitation in a university-sponsored healthcare leadership consortium, which was gathered to assist the university in developing a competencybased healthcare leadership undergraduate program. The participants were all employed by a variety of healthcare organizations located in [state] in the United States. The city was described by one study participant as a "healthcare mecca" (\#14) because of the many healthcare organizations located within the city's regional borders. A snowball sampling technique (Noy, 2008) was used, whereby the first exemplary leaders interviewed were then asked to provide the names and contact information of exemplary leaders in their healthcare organization.

The resulting sample of 26 individuals were identified as effective leaders within the local healthcare industry by peers and fellow leaders. The sample included 16 females and 10 males with a wide range in background, educational experience, and functional area of leadership. In terms of background, participants took a variety of paths to arrive at their present role, including work in the medical field as a nurse, nurse practitioner, doctor, or chaplain, as well as in other arenas such as nonprofit management, information technology, secondary and postsecondary education, human resources, the armed forces, and business. The current position held by a study participant was labeled as "mid-level" (e.g. Manager or Department Director) or "upper-level" (e.g. Senior Vice President or Chief Administrative Officer) by the research team, and 13 participants were assigned to each category. Education levels ranged from an associate's degree to various terminal degrees, including the attainment of a B.S.N., M.S.N, DNP, M.D., MBA, MPA, PhD, or combination thereof, along with other certificates and certifications. While just over one quarter of the sample had only been in their current position for one year or less, the majority of participants had decades of experience in the healthcare industry. The least amount of time spent in the healthcare field was seven years, and four participants had over 30 years of experience. Current functional areas of practice were grouped into six major categories: Clinical Administration and Quality (6), Human Resources (2), Information Technology (2), Nursing Administration (5), Organizational Administration, Strategy, and/or Quality (10), and Technology Administration (2). With the wide array of experiences and organizational roles and affiliations each participant contributed, a variety of perspectives emerged to fuel data analysis. 
Participants were emailed an invitation to participate in the study, noting the healthcare leader who referred them as an exemplary candidate, and requesting an hour to an hour and a half of their time to discuss competencies needed by healthcare leaders. Interviews were scheduled at the convenience of the participants, and participants were also provided with details regarding their research study participation, including discussion of the informed consent form which provided the general purpose of the study, confidentiality parameters, and their permission to record the interview for data collection purposes.

Interview Protocol. In order to answer the research questions, participants were asked open-ended questions without specific reference to any of the NCHL competencies, in order to elicit examples and competencies upper-most in their minds regarding situations most relevant in their current healthcare leadership experiences. This interviewing technique aligns with that recommended for inquiry-based and ethnomethodological interviewing qualitative research (Baker. 2002; Have, 1999). Specific interview questions included the following:

- Describe your career and background, and how you came to be in your current position?

- How would you describe an ideal healthcare leader? Provide an example of someone who you see as epitomizing the ideal healthcare leader.

- Describe an example of a time when you observed an effective or ineffective example of healthcare leadership. What was the situation? What did the leader do that was particularly effective or ineffective? What were the outcomes of this person's behavior?

\section{Results}

In order to address the research question regarding the 26 NCHL competencies (Calhoun et al., 2008) most important in the experiences of the exemplary leaders interviewed in this study, we used NVivo Software (NVivo, 2012) to code the interviews according to the $26 \mathrm{NCHL}$ competencies. Each of the NCHL competencies was organized as a "node," and examples provided by the interviewee that implicitly referred to a competency were coded as that "node."

Table 1 presents the top four of the 26 NCHL competencies in the frequency order with which they were noted, the frequency to which each of the competencies was referred during the interviews, and the dominant competency themes that emerged during the interview data analysis. 
Table 1.

Top Four NCHL Competencies, Themes, and Samples \begin{tabular}{ll}
\hline NCHL Competency/ & Theme(s) and Sample Quotes \\
Frequency of References &
\end{tabular} Change Leadership Consistent Driver and Prioritizer of Change

"an ideal healthcare leader is mission-driven, vision-minded. And keeps the main thing, which for us are the people that we serve. And then figures out how to align systems and inspire the people to pull together to do that."

“...If we have lack of focus, it's hard to move anything along and so I usually try and keep two or three major areas of focus." (\#2)

\section{Understanding the Healthcare Landscape}

"Just by looking at the horizon of where change was going to occur.... knew that at some point, we were going to have to move from a volume-based world to a value-based world. Healthcare, it was fragmented, it was broken.” (\#3)

\begin{tabular}{|c|c|}
\hline Self-Development & $\begin{array}{l}\text { Proactive Continuous Learning } \\
\text { "So not knowing anything about _ , I went off and got trained." } \\
\text { (\#3) } \\
\text { Self-Discovery and Self-Awareness } \\
\text { "I love the diversity here. I love the chance to learn from other } \\
\text { disciplines." } \\
\text { "It's not about what you know, per se. It's about..who you are as } \\
\text { a person. ..It's self-awareness... It's being humble, and...that } \\
\text { humility leads to your ability to listen and to engage other people } \\
\text { and value their ideas. " }(\# 21)\end{array}$ \\
\hline Talent Development & $\begin{array}{l}\text { Moral Leadership Imperative } \\
\text { "...vision is that we really want to foster and develop a system of } \\
\text { problem-solvers... we really try to teach and empower others as } \\
\text { we work with them to develop that skill set." } \\
\text { "You're always developing the next generation of leaders and } \\
\text { helping people to reach their maximum potential. It's really that } \\
\text { important." (\#10) } \\
\text { Coaching with Standards and Compassion } \\
\text { "Take people where they should be and not necessarily where } \\
\text { they want to go" (\#1) }\end{array}$ \\
\hline Team Leadership & $\begin{array}{l}\text { Establishing a Culture of Connection and Purpose } \\
\text { "It's not perfect now but it's a lot better because I'm one of those } \\
\text { people that is a connector." (\#16) } \\
\text { Empowering Delegation } \\
\text { "It's empowering those people who work for you... she really let } \\
\text { us drive the change and create the action plans." } \\
\text { "She leads them, she helps them, she guides them, and she leaves } \\
\text { them alone, right?" (\#04) }\end{array}$ \\
\hline
\end{tabular}


As shown in Table 1, the exemplary leaders in the study described incidents and examples which pertained most frequently to the following NCHL competencies: change leadership, self-development, talent development, and team leadership. These competencies, and the participants' accounts related to these competencies, are described below.

Change Leadership. Change leadership is described by the NCHL competency model as the ability to energize stakeholders and sustain their commitment to changes in approaches, processes, and strategies (Calhoun et al., 2008). This competency was referenced directly and indirectly by all the leaders in this study as an ever-present and underlying focus of their work. They described organization-wide changes, such as mergers and acquisitions, as well as process and personnel changes at the unit and individual levels. Themes that emerged throughout the exemplary leader stories were the following: Change Leadership as a Meta-Competency, The Leader as a Consistent Driver and Prioritizer of Change, and Understanding the Change Landscape. These three themes are discussed below.

Theme: Change Leadership as a Meta-Competency. The change leadership competency could be seen in most leaders' accounts as a "meta-competency" of sorts, in that it provided the context when relating incidents focused on other competencies. Leaders often provided examples of collaboration, problem solving, project management, self-development, talent development, and analytical skills in the context of an effort to drive change; these other competencies were utilized by leaders as strategies or tools in their change initiatives at individual, group, and organizational levels. For example, one interviewee (\#10) discussed the need for IT and meaningful-use measures and electronic health records to be in place as a foundation for change. Another interviewee discussed the need for multidisciplinary teams, forums, and learning approaches to be able to take a strategic perspective and proactively lead change in the organization. The context provided for this need was to maintain a competitive advantage (and avoid bankruptcy and other survival failures), and be able to "stay afloat" in today's ever-changing environment. The following excerpt below pertains to one leader's description of the processes he used - which can be seen to encompass a variety of competencies - to lead an integration project in his organization:

(The) mechanics of change management (begin with an) understanding of what role people need to play, and who are those key players, in the process. And... so, for example, if you're building a steering committee, who are the decision makers, and who has enough authority inside of the different organizations or different functions to actually not only make decisions, but also have the respect of the people who will follow them as they make their decisions? Who are the leaders inside of those organizations? Then you want to build that type of team. And then, the first thing you have to do is... get past the "We, they," to the "We," in the process. And I've seen companies fail because they get so focused on... "This is the way we do it, we bought you in the process, so you do it our way." And I'm sitting there thinking, "Okay, in many cases that might be the case, it might not always be the case, but how do we approach it a little bit less subtly," and say, "Let's talk about the right outcome here," because at the end of the day, you got to get everybody rolling in the same direction, as you get into it. So, once you begin to identify that, then it's communication, communication, communication, communication, and communication. Because you've got to just keep letting people know, "Hey, this was 
the plan, this is what we're doing," you've got to solicit feedback, so people feel involved in the process, as you get into it. And then, you just have to offer those feedback loops; you've got to be able to react to what's going on. And that's where I think in many cases, the ability to listen and the ability to effectively adjust your communication styles become key for you, and then having empathy. Because you've got to understand depending on what side of the fence you come in from... there's a fear on the other side of the fence on "Do these people-- do I have a job? What is it going to be? Am I going to like working for this company?" So the more you can communicate to the workforce, then the faster they will get on your side of the equation. And then you have to be willing to change. If they have a better solution that makes better sense, then you have to be willing to give away what you're doing and then adapt to what they're doing. And I'll tell you, even though sometimes it's hard emotionally for the person who's in charge of doing that to do, it pays such dividends, because you get to the end result faster, because now people say "See they took what I did in the process, they must respect what we do a little bit." Because a lot of times people feel like they've just been disrespected through all these processes. So I think that's how you have to manage change, from a personal level,... (because) this is all around emotion and people. (\#14)

Theme: The Leader as a Consistent Driver and Prioritizer of Change. Leaders spoke of having multiple and expanding priorities, with new laws, policies, and processes being enacted on a continuous basis by their organizations in order to remain responsive and competitive in today's healthcare environment. While acknowledging the need to be responsive and competitive as an organization, the leaders in this study also acknowledged the healthcare leader's critical role in deliberately making known to organizational members which initiatives were top priority and deserving of the most emphasis when deciding on what to focus on a daily basis. As one leader stated:

We had a recent dramatic change in leadership here..., and I feel like there's got to be a clear objective. Now, I'm not saying you can't have a to-do list a mile long. You can have a to-do list a mile long, but something distinct has to be at the top of your to-do list. (\#16)

Within this theme there was also a recognition that leaders used a variety of tools and strategies to drive change. Building relationships across organizational units and functions and understanding various stakeholders' perspectives (e.g. "finding your key stakeholders that you know are already on board with it, and using those individuals as your champions...making them part of the team to champion the change" \#17), developing talent and building employees' skills to handle new changes, and using team leadership were all mentioned as strategies for prioritizing change. As the same leader above stated:

I'm more like a map, where you can... see these are the challenges that are coming ahead, and this is the direction I want us to go in, and you can... see how the path we're taking is to the exclusion of other paths we might have taken. (\#16)

Theme: Understanding the Healthcare Landscape. Leaders described drastic changes that were taking place on an almost-daily basis in their organizations and generally in the healthcare environment. These changes included the need for new processes relating to 
requirements derived from the Affordable Care Act, the need for greater efficiencies given increased competition and scarcer resources, and the need for new ways of looking at healthcare problems in order to find innovative solutions that the organizational systems could bear. The excerpt below is representative of the leaders' constant references to the theme of understanding the changing healthcare environment and the metaphors used to describe this competency:

...The nation's gone through such a massive change...this whole paradigm. So here we are today. We're standing right here, and this is the volume world. This is the value world. Well, we're trying to figure out how do you close this gap? We've got to go from here to here. So value, volume, we've got to make that leap, and we need to build small little steps to get over there, and then slowly fill these in so that way, you can walk right over to it. These are all the planning pieces we need to be doing right now. (\#3)

Self-Development. Self-Development is a competency defined by NCHL as having selfawareness of one's strengths and challenges, and using self-directed learning to address one's challenges (Calhoun et al., 2008). This competency category was referenced directly or indirectly by every participant in the study when describing their own performance as well as when providing examples of effective or ineffective healthcare leadership performance. Themes included proactive continuous learning and self-discovery.

Theme: Proactive Continuous Learning. Participants often referred to both the joy and necessity for continuous learning in their jobs and careers, and described numerous instances when they would proactively seek avenues for learning - through self-study, discussions and collaborations with experts across and outside the organization (e.g. "learning the nooks and crannies of the system" (\#19)), and challenging job assignments. Study participants often referred to changes in their job situations as "learning opportunities," and described in detail what they learned when facing changes and challenges. One leader described needing to be "an avid learner because of how fast healthcare is changing" (\#10). Likewise, many participants described a deliberate career management journey, where they looked for gaps in their skills and knowledge sets, and proactively worked to address these gaps by volunteering for new and challenging assignments. As one leader stated:

So not knowing anything about hyperbarics, I went off and got trained, and became a Hyperbaric Tech, because I thought if I'm going to run a department, I need to know how it works. I need to know the equipment, the safeties and the risks. And just overall protocols. And in order to lead a team, I had to know what they did, what it comprised of. So I went off and went to _ Medical Center in _ _ , and spent a week out there with their team. And they actually had a training program, so I came back certified. (\#3)

Another leader succinctly summarized his continuous learning journey as building a pyramid of skills throughout his varied career in healthcare:

..Education. At this level right here, you want as wide a base of your pyramid as possible 'cause you never know what you're gonna need as you go up. (\#19) 
Theme: Self-Discovery and Self-A wareness. Self-awareness was a key component of this drive for self-development; nearly all participants alluded to a quest for self-awareness which they used as a competency in performing their job. As one leader stated:

When somebody asks me to do something, I say, "You know what? Two of those match up with my strengths. So, yeah, I think I could bring that home. Let me go find my-- X person on my team that has this skill set and, between the two of us, sure, we can knock that out. And we can meet that timeframe. (\#1)

In discussing the importance of self-awareness, another leader described self-acceptance and resilience in the face of understanding one's strengths and shortcomings:

We all have our baggage. That's right... and so it's not the elimination of baggage. It's just knowing what my baggage is and going, "This feels familiar and when I'm in this situation this tends to be what I do"... I think you're raising their awareness, but more than that, you're inserting in the wise person that there's a process for becoming and staying aware. (\#19)

Talent Development. Talent development is described in the NCHL competency model as building the organization's talent pool by recognizing and supporting the development of employees' skills and knowledge with coaching, feedback, and assignment activities (Calhoun et al., 2008). As was found for the self-development and change leadership competency categories, the exemplary leaders interviewed in this study referenced multiple examples which alluded to the talent development competency. Many leaders exhibited a sense of urgency and clear ownership of talent development as a leadership responsibility, both for the organization's needs as well as for their employees' growth. Two themes that emerged within this competency include: moral leadership imperative and coaching with standards and compassion.

Theme: Moral Leadership Imperative. A common theme among leaders in the study was a sense of "moral obligation" to sincerely care about employees' development, as referenced by one leader:

I said, "We have a moral obligation....We as an organization have a moral obligation to this group of employees to ensure that there's nothing that the company's doing intentionally or unintentionally to block their advancement." So for example, if I want to go learn about Excel, I go over and I take a class...I'm still getting paid for that. Environmental Service worker wants to come to our program, they got to clock out. Well, we fixed that so they're on the clock. We keep them whole. So we're affording them some education, some coaching, some things on the clock in a way that's going to help them... What we said is we're not going to drag anybody across the finish line. But we're going to make a clear path to the finish line. We're going to clear the path to the finish line. But ultimately, you have to take your own exams, write your own papers. Everybody. But by golly, I don't want anybody leaving here thinking they couldn't succeed because of something that we did or didn't do (\#19). 
Theme \#2: Coaching with Standards and Compassion. Several leaders described a strong caring and compassion component related to their coaching efforts and self-identity as a leader, as noted by the following:

We need more educated healthcare leaders. I mean, it's hard because I know we've got 160 medical practices out there that have managers at different skillsets. Some of them are high school graduates that were on-the-job trained. We've got some that have associate degrees. We've got some that are bachelor degreed. So we've got a very variable group of people up there that are leading our medical groups. Some are very successful and some have struggled. I think there needs to be a program that brings them up to at least a standard of healthcare leadership and prepares them for what the future holds for healthcare (\#18).

Team Leadership. The team leadership competency is defined in the NCHL model as managing team meetings, communicating with team members, establishing goals and positive norms as the leader of a team, and keeping the team informed and engaged (Calhoun et al., 2008). In this study, nearly all participants provided examples that pertained to aspects of the team leadership competency. Two of the major themes which emerged during the interview conversations pertained to empowering delegation and establishing a culture of connection and purpose.

Theme: Empowering Delegation. Leaders referenced the importance of delegation in the context of the constant need to drive change, ever-increasing workloads, and the need to identify and develop their high-performing team members. Delegation was also seen as a way to gain buy-in and engagement for an overall change goal, e.g.:

As a leader I think that's very important, not only to hear what they were saying but act on it. And we hired the person that they thought was appropriate and she's doing phenomenally, so it's good to get their buy-in. (\#07).

You can order things and, when you order people to do things, I think you're gonna get exactly what you order. But, if you can persuade them, bring their heart into it and understand, then you've got a much bigger commitment from them (\#19).

Theme: Establishing a Culture of Connection and Purpose: Leaders discussed the need to create a positive team culture where everyone understands their role and its connection to a greater purpose. To create a positive team culture, leaders spoke of recognizing and rewarding effective performance, being available for their subordinates, and being positive role models for others.

So I have a vice president here that-- she's the vice president of quality, which you wouldn't think would be someone who's terribly engaged. To me that sounds like you're looking at data. She's very, very engaged in her managers, her abstracters. She reaches out to people weekly, so that rounding of, "I'm walking through your department just to say hey and, you know, gosh, how are things today," or "You guys look really busy." It could be a five-minute conversation but it means the world that they're available. 
Availability is a very big thing, especially for higher-level leaders. I think sometimes there's a disconnect with that. (\#07)

\section{Discussion}

Results from the present study suggest that the exemplary healthcare leaders interviewed in this study perceive that change leadership, self-development, talent development, and team leadership are the four most critical competencies for effective performance of their healthcare leadership roles. In particular, the change leadership competency appeared to serve as a "metacompetency" encompassing the other competencies as tools or strategies in service to the constant change leadership required in today's healthcare leaderenvironment.

The fact that the participants in the study were perceived and identified by their C-level managers as exemplary leaders is an important consideration in interpreting the results from the study. By definition, these leaders are successful and effective in navigating the requirements of their healthcare leader roles. As such, their insights into the competencies most needed for fulfilling healthcare leadership role requirements can be given more credence than if a more random sample was interviewed, which may have included leaders who are less successful or not exemplary in their role behaviors. Likewise, however, it may be that more "average" or less exemplary leaders may identify other competencies as being even more critical for them. It is suggested, therefore, that future studies explore the experiences of a wider range of healthcare leaders to gain a broader understanding of their lived experiences in today's healthcare environment.

The qualitative methodology used in the present study is particularly appropriate for the given research goal of exploring healthcare leaders' accounts of their actions in a variety of healthcare leadership situations. Interviews of exemplary healthcare leaders allow for in-depth delving and follow-up questions pertaining to the underlying meaning of competencies and examples pertaining to those competencies. This type of depth of questioning and meaningmaking is not possible with quantitative survey analysis. On the other hand, quantitative studies are suggested to provide insights relating to healthcare leaders' ratings of the competencies most needed for their work, and to also provide greater generalizability of study findings.

It is noteworthy that findings from the current study suggest that "people"-related competencies, and in particular competencies related to talent development and selfdevelopment, were overwhelmingly the most-referred-to competencies by the exemplary leaders in this study. This finding, in conjunction with the plethora of studies alluding to the changing nature of the healthcare environment and the increasing responsibilities of the healthcare leader role, suggests that a desire and aptitude for continuous learning may serve as a critical foundational competency for current and future healthcare leaders. These findings are also aligned with recent research suggesting that the emotional intelligence competencies - related to the "soft skills" found in this study - are most correlated with healthcare leaders' performance (Weiszbrod, 2015). Taken together, results suggest that future research should investigate healthcare leader talent development and educational needs and opportunities. 
Areas for future research may also include needs assessment studies of leaders at all position and performance levels in order to identify which competencies represent a gap between actual and ideal readiness for handling the challenges in today's healthcare environment. Needs assessment studies could investigate both 360 performance ratings and self-report qualitative interviews with leaders and in relation to objective measures important for healthcare organizational performance such as mortality rates, readmission rates, HCAHPS survey scores, and HQA process measures. Findings from future studies, in conjunction with this study, may suggest areas of emphasis in educational and development curriculum design and updates.

Leadership education challenges suggested by this study include knowledge versus skill development of the leadership competencies identified as most prominent in healthcare leaders' accounts of effectiveness in the current healthcare environment: change leadership, self development, talent development, and team leadership. Leadership education programs which focus primarily on knowledge components needed for healthcare leadership (e.g. which focus on increasing students' knowledge of principles of finance, organizational change, human resource management, quality measures, and other knowledge-related principles) may need to be augmented with additional competency development activities that build the behavioral components of leadership competencies upon which the exemplary leaders in this study focused as being keys to their success. Competency-based education (CBE) programs, which require students to exhibit competency in a focal course curriculum objective before moving on to another course in the program, are becoming increasingly prominent in higher education, and may provide a promising venue for leadership competency development (Gruppen, Mangrulkar, \& Kolars, 2012; Smith \& Wolverton, 2010; Ordonez, 2014). Regardless of whether a healthcare leadership education program is more traditionally-designed or competency-based, results from the present study suggest that interpersonal and self-management skills are critical components of the curriculum. These types of leadership skills pose challenges for development (Weiszbrod, 2015), and additional research is needed to explore educational strategies such as leadership assessment center methodology (Amogoh, 2010; Eurich, Krause, Cigularov, and Thornton, 2009) and other behaviorally-focused leadership development tools for developing these competencies.

Engaged, passionate, visionary, caring, understanding of subordinates' individual needs all of these descriptors characterize the healthcare leaders in this study as well as the effective leaders they described in healthcare settings. It would therefore be fruitful for future research to use the lens of leadership theories - and in particular, authentic leadership and transformational leadership theory, in light of the participants' emphasis on change leadership and selfdevelopment - to analyze the underlying influence processes most used by effective healthcare leaders in today's healthcare industry environment.

\section{Conclusion}

This interview study provides insights regarding the competencies perceived by a sample of current exemplary healthcare leaders as most critical for the effective performance of their leadership roles. Future research using both qualitative and quantitative methodologies to assist in ascertaining specific skills embedded within the broader competency domains are recommended to meet the changing development and education needs of healthcare leaders in 
today's environment. In light of the current study's findings pertaining to the importance of healthcare leadership competency development, the findings from this and future studies have critically important implications for talent identification, selection and assessment, development, and retention of healthcare leaders as well as the learning functions of healthcare organizations.

\section{References}

Al-Sawai, A. (2013). Leadership of healthcare professionals: where do we stand? OmanMedical Journal, 28(4), 285.

Amagoh, F. (2009). Leadership development and leadership effectiveness. Management Decision, 47(6), 989-999.

Baker, C. D. (2002). Ethnomethodological analyses of interviews. In J. F. Gubrium \& J. A. Holstein (Eds.), Handbook of interviewing: Context and method (pp. 777-795). Thousand Oaks, CA: Sage.

Berg, B. L., Lune, H., \& Lune, H. (2004). Qualitative research methods for the social sciences (Vol. 5): Pearson Boston, MA.

Boyatzis, R. (2007). Review of literature on competency model development. The Indian CEO: A Portrait of Excellence, edited by SM Spencer, T. Rahah, SA Narayan, S. Mohan, and G. Lahiri. Thousand Oaks, CA: Sage Publications.

Boyatzis, R. E. (1982). The competent manager: A model for effective performance: John Wiley \& Sons.

Boyatzis, R. E. (2006). Using tipping points of emotional intelligence and cognitive competencies to predict financial performance of leaders. Psicothema, 18(Suplemento), 124-131.

Boyatzis, R. E., Leonard, D., Rhee, K., \& Wheeler, J. V. (1996). Competencies can be developed, but not in the way we thought. Capability, 2(2), 25-41.

Calhoun, J. G., Davidson, P. L., Sinioris, M. E., Vincent, E. T., \& Griffith, J. R. (2002). Toward an understanding of competency identification and assessment in health care management. Quality Management in Healthcare, 11(1), 14-38.

Calhoun, J. G., Dollett, L., Sinioris, M. E., Wainio, J. A., Butler, P. W., Griffith, J. R., \& Warden, G. L. (2008). Development of an interprofessional competency model for healthcare leadership. Journal of Healthcare Management, 53(6), 375.

Calhoun, J. G., Vincent, E. T., Calhoun, G. L., \& Brandsen, L. E. (2008). Why competencies in graduate health management and policy education? Journal of Health Administration Education, 25(1), 17-35. 
Campion, M. A., Fink, A. A., Ruggeberg, B. J., Carr, L., Phillips, G. M., \& Odman, R. B. (2011). Doing competencies well: Best practices in competency modeling. Personnel Psychology, 64(1), 225-262.

Castells, M. (2000). Information technology and global capitalism. On the Edge: Essays on a Runaway World. London: Jonathan Cape.

Clark, J., \& Armit, K. (2010). Leadership competency for doctors: a framework. Leadership in Health Services, 23(2), 115-129.

DiCiccoDBloom, B., \& Crabtree, B. F. (2006). The qualitative research interview. Medical Education, 40(4), 314-321.

Eurich, T., Krause, D., Cigularov, K., and Thornton, G. III. (2009). Assessment centers: Current practices in the United States. Journal of Business Psychology, 24 (4), 387-407.

Franco, M., \& Almeida, J. (2011). Organisational learning and leadership styles in healthcare organisations: An exploratory case study. Leadership \& Organization Development Journal, 32(8), 782-806.

Fried, B. J., \& Fottler, M. D. (2013). Fundamentals of human resources in healthcare. American College of Healthcare Executives.

Garman, A., \& Scribner, L. (2011). Leading for quality in healthcare: Development and validation of a competency model. Journal of Healthcare Management, 56(6), 373.

Garman, A. N., \& Johnson, M. P. (2006). Leadership competencies: An introduction. Journal of Healthcare Management, 51(1), 13.

Griffith, J. R. (2007). Improving preparation for senior management in healthcare. Journal of Health Administration Education, 24(1), 11-32.

Gruppen, L. D., Mangrulkar, R. S., \& Kolars, J. C. (2012). The promise of competency-based education in the health professions for improving global health. Human Resourcesfor Health, 10(1), 1.

Have, P. T. (1999). Doing conversation analysis: A practical guide. Thousand Oaks, CA: Sage.

Kovner, A. R., \& Rundall, T. G. (2006). Evidence-based management reconsidered. Frontiers of Health Services Management, 22(3), 3.

Kovner, A. R., Wagner, R. F., \& Curtis, R. S. (2001). Better information for the board/Practitioner application. Journal of Healthcare Management, 46(1), 53. 
McAlearney, A. S. (2010). Executive leadership development in US health systems. Journal of Healthcare Management, 55(3), 206-222.

National Center for Healthcare Leadership (NCHL) (2004). Healthcare leadership competency model: Summary. Retrieved from http://www.nchl.org/Documents/NavLink/Competency Model-summary_uid31020101024281.pdf

Noy, C. (2008). Sampling knowledge: The hermeneutics of snowball sampling in qualitative research. International Journal of Social Research Methodology, 11(4), 327-344.

Ordonez, B. (2014). CompetencyDbased education: Changing the traditional college degree power, policy, and practice. New Horizons in Adult Education and Human Resource Development, 26(4), 47-53.

Patterson, T. E., Champion, H., Browning, H., Torain, D., Harrison, C., Gurvis, J., Fleenor, J., \& Campbell, M. (2011). Addressing the leadership gap in healthcare: What's needed when it comes to leader talent. Retrieved from http://insights.ccl.org/wpcontent/uploads/2015/04/addressingLeadershipGapHealthcare.pdf

Schön, D. A. (1991). The reflective turn: Case studies in and on educational practice: Teachers College Press: New York.

Senge, P. M. (1990). The art and practice of the learning organization: New York: Doubleday.

Shippmann, J. S., Ash, R. A., Batjtsta, M., Carr, L., Eyde, L. D., Hesketh, B., Sanchez, J. I. (2000). The practice of competency modeling. Personnel Psychology, 53(3), 703-740.

Smith, Z. A., \& Wolverton, M. (2010). Higher education leadership competencies: Quantitatively refining a qualitative model. Journal of Leadership \& Organizational Studies, 17(1), 61-70.

Stefl, M. E., \& Bontempo, C. (2008). Common competencies for all healthcare managers: The healthcare leadership alliance model. Journal of Healthcare Management, 53(6), 360.

Weiszbrod, T. (2015). Health care leader competencies and the relevance of emotional intelligence. The Health Care Manager, 34(2), 140-146.

White, K. R., \& Griffith, J. R. (2016). The well-managed healthcare organization. Arlington, VA: AUPHA Health Administration Press. 


\section{Author Biographies}

Dr. Ann Herd is an Assistant Professor of Human Resources and Organization Development in the Organizational Leadership and Learning program at the University of Louisville. She researches, teaches, and regularly provides services to industry and the military in the areas of leadership assessment and development, executive coaching, military career transitioning, women and leadership, and healthcare and global leadership competency development.

Dr. Brittany Adams-Pope is an Assistant Professor in the Equine Industry Program in the College of Business at the University of Louisville. She received her Ph.D. in Agricultural Leadership at the University of Florida in 2013. Her research interests include authentic leadership, leadership development, equine-assisted education and activites, and issues in the equine industry.

Amanda Bowers is a Ph.D. student in the Educational Leadership, Evaluation, and Organizational Development Program at the University of Louisville, studying Higher Education. She received her M.Ed. from Vanderbilt University's Peabody College of Education and Human Development in Community Development and Action, and B.S. in Political Science and Human and Organizational Development from Vanderbilt University.

Brittany Sims is a Graduate Research Assistant in the Equine Industry Department and MBA student at the University of Louisville. Her research interests include volunteer management, leadership, and community and economic development, and she plans to pursue a career in public administration. 